\title{
Vibration Analysis of Non-homogenous Orthotropic Visco-elastic Rectangular Plate of Parabolically Varying Thickness with Thermal Effect
}

\author{
Pooja Singhal \\ Department of Mathematics, P.C.E.T., Lalru- 140501, Punjab, India \\ Arun Kumar Gupta \\ Department of Mathematics, M.S. College, Saharanpur- 247001, UP, India
}

\begin{abstract}
(Received 25 March 2014; accepted 25 August 2015)
The present work analyses the vibration behaviour of non-homogeneous orthotropic visco-elastic rectangular plate of parabolically varying thickness on the basis of classical plate theory when the all edges are clamped and are subjected to linearly thermal variation. For non-homogeneity of the plate material it is assumed that the density of the plate material varies parabolically along the x-direction. For visco-elastic materials, basic elastic and viscous elements are combined. The Kelvin model for visco-elasticity is considered here, which is a combination of elastic and viscous elements connected in parallel. Using the separation of variable method, the governing differential equation has been solved. The time period and deflection corresponding to the first two modes of vibrations of clamped plates have been calculated for different values of thermal gradients, non-homogeneity constants, taper constants, and aspect ratio, with the help of Rayleigh-Ritz techniques, and are shown by graphs.
\end{abstract}

\section{INTRODUCTION}

The thermal effect of non-homogenous viscoelastic plates on vibration is of great interest in the field of engineering, with applications such as improved designing of gas turbines, jet engines, space craft, and nuclear power projects, where metals and their alloys exhibit visco-elastic behaviour. Therefore, for these reasons, such structures are exposed to high-intensity heat fluxes, and thus the material properties undergo significant changes. In particular, the thermal effect of elasticity of the material on the modules cannot be taken as negligible.

Space technology is developing very rapidly in the present era, and the importance of studying the vibration of plates of certain aspect ratios with some simple restraints on the boundaries has increased. The motors of rockets and aircraft in cold regions are developed with the use of soft filaments in aerospace structure supported with elastic or visco-elastic media. When finalising a design, a construction engineer should understand the first few modes of vibration, as they are significant.

Plates of variable thickness have been extensively used in civil, electronic, mechanical, aerospace, and marine engineering applications. The practical importance of such plates has made vibration analysis essential, especially since the vibratory response needs to be accurately determined in the design process in order to avoid resonance excited by internal or external forces.

The plate type's structural components in aircraft and rockets have to operate under elevated temperatures that cause nonhomogeneity in the plate material, i.e. elastic constants of the materials become functions of space variables. An up-to-date survey of the research in this area shows that authors have come across various models to account for non-homogeneity of plate materials, and many researchers have proposed dealing with vibration. However, none of them consider nonhomogeneity with a thermal effect on orthotropic visco-elastic plates of parabolically varying thickness.

The term vibration describes repetitive motion that can be measured and observed in a structure. Unwanted vibration can cause fatigue or degrade the performance of the structure. Therefore, it is desirable to eliminate or reduce the effects of vibration. In other cases, the goal may be to understand the effect of vibration on the structure, to control or modify the vibration, or to isolate it from the structure and minimise the structural responses.

Vibration can be sub-categorised, such as free versus forced vibration, sinusoidal versus, and linear versus rotation-induced vibration. Free vibration is the natural response of a structure to some impact or displacement. The response is completely determined by the properties of the structure, and its vibration can be understood by examining the structures mechanical properties. For example, when we pluck the string of a guitar, it vibrates at the tuned frequency and generates the desired sound. The frequency of the tone is a function of the tension in string and is not related to the plucking technique.

A great deal of research informs the study presented here. Laura, et al. discussed transverse vibrations of rectangular plates with thickness varying in two directions and with edges elastically restrained against rotation. ${ }^{1}$ Leissas monograph ${ }^{2}$ contains an excellent discussion of the subject of vibrating plates with elastic edge support. Gupta and Singhal discussed the effect of non-homogeneity on the thermally-induced vibration of an orthotropic visco-elastic rectangular plate of linearly varying thickness. ${ }^{3}$ Lal has studied the transverse vibra- 
tions of orthotropic non-uniform rectangular plates with continuously varying density. ${ }^{4}$ Sobotka examined the free vibration of visco-elastic orthotropic rectangular plates. ${ }^{5}$ Singh and Saxena analysed the transverse vibration of rectangular plates with bi-directional thickness variation. ${ }^{6}$ Bambill, et al. studied the transverse vibrations of an orthotropic rectangular plate of linearly varying thickness and a free edge. ${ }^{7}$ Tomar and Gupta solved the vibration problem of an orthotropic rectangular plate of varying thickness subjected to a thermal gradient. $^{8-10}$

Gupta, et al. discussed the vibration of non-homogeneous circular plate of nonlinear thickness variation by a quadrature method. ${ }^{11}$ Gupta and Kumar studied the effect of exponential temperature variation on vibration of orthotropic rectangular plate with linearly thickness variation in both directions. ${ }^{12}$ Gupta, et al. solved the problem of thermal effect on vibration of non-homogeneous orthotropic rectangular plate having bi-directional parabolically varying thickness. ${ }^{13}$ Gupta and Khanna discussed vibration of viscoelastic rectangular plate with linearly thickness variations in both directions. ${ }^{14}$ Laura and Gutierrez discussed vibration analysis of a rectangular plate subjected to a thermal gradient. ${ }^{15}$ Gupta and Khanna solved the problem of vibrations of clamped visco-elastic rectangular plate with parabolic variable thickness. ${ }^{16}$ Effect of thermal gradient on free vibration of clamped visco elastic plate was discussed by Gupta and Kaur. ${ }^{17}$ Finally, Gupta and Kumar studied the thermal effect on vibration of orthotropic rectangular plate with parabolic thickness variations. ${ }^{18}$

The analysis presented in this chapter studies the effect of parabolic non-homogeneity on thermally-induced vibration of an orthotropic visco-elastic rectangular plate of parabolically varying thickness. It is clamp-supported on all four edges. The assumption of small deflection and linear orthotropic viscoelastic properties are made. It is further assumed that the viscoelastic properties of the plate are of the Kelvin type. The time period and deflection for the first two modes of vibration are calculated for the various values of thermal constants, nonhomogeneity constants, aspect ratio, and taper constants. The results are shown graphically.

\section{ANALYSIS}

The equation of motion of a visco-elastic rectangular plate of variable thickness is as follows: ${ }^{3}$

$$
\begin{aligned}
& {\left[D_{x} \frac{\partial^{4} W}{\partial x^{4}}+D_{y} \frac{\partial^{4} W}{\partial y^{4}}+2 H \frac{\partial^{4} W}{\partial x^{2} \partial y^{2}}+2 \frac{\partial H}{\partial x} \frac{\partial^{3} W}{\partial x \partial y^{2}}\right.} \\
& +2 \frac{\partial H}{\partial y} \frac{\partial^{3} W}{\partial x^{2} \partial y}+2 \frac{\partial D_{x}}{\partial x} \frac{\partial^{3} W}{\partial x^{3}}+2 \frac{\partial D_{y}}{\partial y} \frac{\partial^{3} W}{\partial y^{3}}+\frac{\partial^{2} D_{x}}{\partial x^{2}} \frac{\partial^{2} W}{\partial x^{2}} \\
& \left.+\frac{\partial^{2} D_{y}}{\partial y^{2}} \frac{\partial^{2} W}{\partial y^{2}}+\frac{\partial^{2} D_{1}^{\prime}}{\partial x^{2}} \frac{\partial^{2} W}{\partial y^{2}}+\frac{\partial^{2} D_{1}^{\prime}}{\partial y^{2}} \frac{\partial^{2} W}{\partial x^{2}}+4 \frac{\partial^{2} D_{x y}}{\partial x \partial y} \frac{\partial^{2} W}{\partial x \partial y}\right] \\
& -\rho h p^{2} W=0
\end{aligned}
$$

and

$$
\ddot{T}+p^{2} \tilde{D} T=0
$$

where Eqs. 1 and 2 are the differential equations of motion for an orthotropic plate of variable thickness, and the time function for visco-elastic orthotropic plate for free vibration, respectively. Here, $p^{2}$ is a constant, and $H=D_{1}^{\prime}+2 D_{x y}$,
$D_{x}=\frac{E_{x} h^{3}}{12\left(1-v_{x} v_{y}\right)}$, is called the flexural rigidity of the plate in $\mathrm{X}$-direction,

$D_{y}=\frac{E_{y} h^{3}}{12\left(1-v_{x} v_{y}\right)}$, is called the flexural rigidity of the plate in y-direction,

$D_{x y}=\frac{G_{x y} h^{3}}{12}$, is called the torsion rigidity,

$D_{1}^{\prime}=v_{x} D_{y}\left(=v_{y} D_{x}\right), \tilde{D}$ is the Rheological operator, $E_{x}$ and $E_{y}$ are the modules of elasticity in x- and y-directions, respectively, $v_{x}$ and $v_{y}$ are the Poisson ratios, and $G_{x y}$ is the shear modulus.

The study assumes steady, one-dimensional temperature distribution along the length, i.e. the $\mathrm{x}$-direction, for the plate as

$$
\tau=\tau_{0}\left(1-\frac{x}{a}\right)
$$

where $\tau$ denotes the temperature excess above the reference temperature at any point at distance $\frac{x}{a}$, and $\tau_{0}$ denotes the temperature excess above the reference temperature at the end, i.e. $x=a$.

The temperature dependence of the modulus of elasticity for most engineering materials can be expressed in the following form:

$$
\left.\begin{array}{l}
E_{x}=E_{1}(1-\gamma \tau), \\
E_{y}=E_{2}(1-\gamma \tau), \\
G_{x y}=G_{0}(1-\gamma \tau)
\end{array}\right\}
$$

Here, $E_{1}$ and $E_{2}$ are values of the Youngs moduli, respectively, along the $\mathrm{x}$ - and $\mathrm{y}$-axis at the reference temperature, i.e. at $\tau=0$, and $\gamma$ is the slope of the variation of the modulus of elasticity with $\tau$.

Thus, the modulus variation becomes

$$
\left.\begin{array}{l}
E_{x}(x)=E_{1}[1-\alpha(1-x / a)], \\
E_{y}(x)=E_{2}[1-\alpha(1-x / a)], \\
G_{x y}(x)=G_{0}[1-\alpha(1-x / a)]
\end{array}\right\}
$$

where $\alpha=\gamma \tau_{0}(0 \leq \alpha<1)$, a parameter known as thermal gradient.

The expression for the strain energy $V$ and kinetic energy $P$ in the plate are: ${ }^{2}$

$$
\begin{gathered}
V=\frac{1}{2} \int_{0}^{a} \int_{0}^{b}\left[D_{x}\left(W_{, x x}\right)^{2}+D_{y}\left(W_{, y y}\right)^{2}\right. \\
\left.+2 D_{1} W_{, x x} W_{, y y}+4 D_{x y}\left(W_{, x y}\right)^{2}\right] d x d y \\
\mathrm{P}=\frac{1}{2} p^{2} \int_{0}^{a} \int_{0}^{b} \rho h W^{2} d x d y .
\end{gathered}
$$

This study assumes that the thickness and density both vary parabolically in the $\mathrm{x}$-direction, respectively; therefore, one can take

$$
h=h_{0}\left\{1+\beta(x / a)^{2}\right\}
$$

and

$$
\rho=\rho_{0}\left\{1+\alpha_{1}(x / a)^{2}\right\}
$$

where $\beta$ is the taper constant and $\alpha_{1}$ is the non-homogeneity constant. 


\section{SOLUTION AND FREQUENCY EQUATION}

The Rayleigh-Ritz technique has been utilised here for finding the solution. This method requires that the maximum strain energy must be equal to the maximum kinetic energy. So, it is necessary for the problem under consideration that

$$
\delta(V-P)=0
$$

for arbitrary variations of $W$, satisfying the relevant geometrical boundary conditions, which are

$$
\begin{aligned}
& W=W_{, x}=0 \quad \text { at } \quad \mathrm{x}=0, \mathrm{a}, \\
& W=W_{, y}=0 \quad \text { at } \quad \mathrm{y}=0, \mathrm{~b} ;
\end{aligned}
$$

and the corresponding two-term deflection function is taken as follows: ${ }^{3}$

$$
\begin{aligned}
W= & {[(x / a)(y / b)(1-x / a)(1-y / b)]^{2} } \\
& {\left[A_{1}+A_{2}(x / a)(y / b)(1-x / a)(1-y / b)\right] . }
\end{aligned}
$$

The non-dimensional variables are

$$
\begin{aligned}
X & =x / a, \quad Y=y / a, \\
\bar{W} & =W / a, \quad \bar{h}=h / a, \\
\bar{\rho} & =\rho / a \\
E_{1}^{*} & =E_{1} /\left(1-\nu_{x} \nu_{y}\right), \\
E_{2}^{*} & =E_{2} /\left(1-\nu_{x} \nu_{y}\right), \\
E^{*} & =\nu_{x} E_{2} *=\nu_{y} E_{1}{ }^{*} .
\end{aligned}
$$

By using Eqs. 5, 8, 9, and 13 in Eqs. 6 and 7, one gets

$$
P=\frac{1}{2} \rho_{o} p^{2} \overline{h_{o}} a^{5} \int_{0}^{1} \int_{0}^{b / a}\left[\left(1+\alpha_{1} X^{2}\right)\left(1+\beta X^{2}\right) \bar{W}^{2}\right] d X d Y
$$

and

$$
\begin{aligned}
V & =R \int_{0}^{1} \int_{0}^{b / a}\left[\{ 1 - \alpha ( 1 - X ) \} ( 1 + \beta X ^ { 2 } ) ^ { 3 } \left\{\left(\bar{W}_{, X X}\right)^{2}\right.\right. \\
& +\left(E_{2}^{*} / E_{1}^{*}\right)\left(\bar{W}_{, Y Y}\right)^{2}+\left(2 E^{*} / E_{1}^{*}\right) \bar{W}_{, X X} \bar{W}_{, Y Y} \\
& \left.\left.+\left(4 G_{o} / E_{1}^{*}\right)\left(\bar{W}_{, X Y}\right)^{2}\right\}\right] d X d Y
\end{aligned}
$$

where

$$
R=\frac{1}{2}\left(E_{1} *{\overline{h_{o}}}^{3} / 12\right) a .
$$

Upon substituting the values of $\mathrm{P}$ and $\mathrm{V}$ from Eqs. 14 and 15 into Eq. 10, we get

$$
\begin{gathered}
\left(V_{1}-\lambda^{2} p^{2} P_{1}\right)=0 \\
V_{1}=\int_{0}^{1} \int_{0}^{b / a}\left[\{ 1 - \alpha ( 1 - X ) \} ( 1 + \beta X ^ { 2 } ) ^ { 3 } \left\{\left(\bar{W}_{, X X}\right)^{2}\right.\right. \\
+\left(E_{2}{ }^{*} / E_{1}{ }^{*}\right)\left(\bar{W}_{, Y Y}\right)^{2}+\left(2 E^{*} / E_{1}{ }^{*}\right) \bar{W}_{, X X} \bar{W}_{, Y Y} \\
\left.\left.+\left(4 G_{o} / E_{1}^{*}\right)\left(\bar{W}_{, X Y}\right)^{2}\right\}\right] d X d Y
\end{gathered}
$$

and

$$
\left.P_{1}=\int_{0}^{1} \int_{0}^{b / a}\left[\left\{1+\alpha_{1} X^{2}\right)\right\}\left(1+\beta X^{2}\right) \bar{W}^{2}\right] d X d Y
$$

where

$$
\lambda^{2}=\frac{12 a^{4} \rho_{o}}{E_{1}{ }^{*} \bar{h}_{o}^{2}} .
$$

Eq. 17 involves the unknowns $A_{1}$ and $A_{2}$, arising due to the substitution of $W(x, y)$ from Eq. 12. These two constants are to be determined from Eq. 17, as follows:

$$
\frac{\partial}{\partial A_{n}}\left(V_{1}-\lambda^{2} p^{2} P_{1}\right)=0, \quad \text { where } \quad \mathrm{n}=1,2 .
$$

Upon simplifying Eq. 21 we get

$$
b_{n 1} A_{1}+b_{n 2} A_{2}=0
$$

where $n=1,2$, and $b_{n 1}, b_{n 2}$ involves parametric constants and the frequency parameter $p$. For a non-trivial solution, the determinant of the coefficient of Eq. 22 must be zero. So, we get the frequency as follows:

$$
\left|\begin{array}{ll}
b_{11} & b_{12} \\
b_{21} & b_{22}
\end{array}\right|=0
$$

Upon solving Eq. 23, one gets a quadratic equation in $p^{2}$, which gives two values of $p^{2}$. Upon substituting the value of $A_{1}=1$, by choice, into Eq. 12 , one gets $A_{2}=-b_{11} / b_{12}$, and hence $W$ becomes

$$
\begin{aligned}
W= & {\left[X Y \frac{a}{b}(1-X)\left(1-Y \frac{a}{b}\right)\right]^{2} } \\
& {\left[1+\left(-\frac{b_{11}}{b_{12}}\right) X Y\left(\frac{a}{b}\right)(1-X)\left(1-Y \frac{a}{b}\right)\right] . }
\end{aligned}
$$

\section{TIME FUNCTION OF VIBRATION OF VISCO-ELASTIC PLATES}

The expression for the time function of free vibrations of visco-elastic plates of variable thickness can be derived from Eq. 2, which depends upon the visco-elastic operator $\tilde{D}$, and which for Kelvins Model, can be taken as follows:

$$
\tilde{D} \equiv\left\{1+\left(\frac{\eta}{G}\right)\left(\frac{d}{d t}\right)\right\} ;
$$

where $\eta$ is the visco-elastic constant and $\mathrm{G}$ is the shear modulus. Assuming that the temperature dependence of the viscoelastic constant $\eta$ and the shear modulus $G$ are in the same form as that of Youngs moduli, we have

$$
G(\tau)=G_{0}\left(1-\gamma_{1} \tau\right), \quad \eta(\tau)=\eta_{0}\left(1-\gamma_{2} \tau\right)
$$

where $G_{0}$ is the shear modulus, and $\eta_{0}$ is the visco-elastic constant at some reference temperature, i.e. at $\tau=0, \gamma_{1}$ and $\gamma_{2}$ are the slope variation of $\tau$ with $G$ and $\eta$, respectively. Substituting the value of $\tau$ from Eq. 3, and using Eq. 13 in Eq. 26, one gets the following:

$$
\begin{aligned}
G & =G_{0}\left[1-\alpha_{5}(1-X)\right], \quad \text { where } \quad \alpha_{5}=\gamma_{1} \tau_{0}, 0 \leq \alpha_{5}<1 \\
\eta & =\eta_{0}\left[1-\alpha_{4}(1-X)\right], \quad \text { where } \quad \alpha_{4}=\gamma_{2} \tau_{0}, 0 \leq \alpha_{4}<1 .
\end{aligned}
$$

Here, $\alpha_{4}$ and $\alpha_{5}$ are thermal constants.

After using Eq. 25 in Eq. 2, one obtains the following:

$$
\ddot{T}+p^{2} k \dot{T}+p^{2} T=0
$$




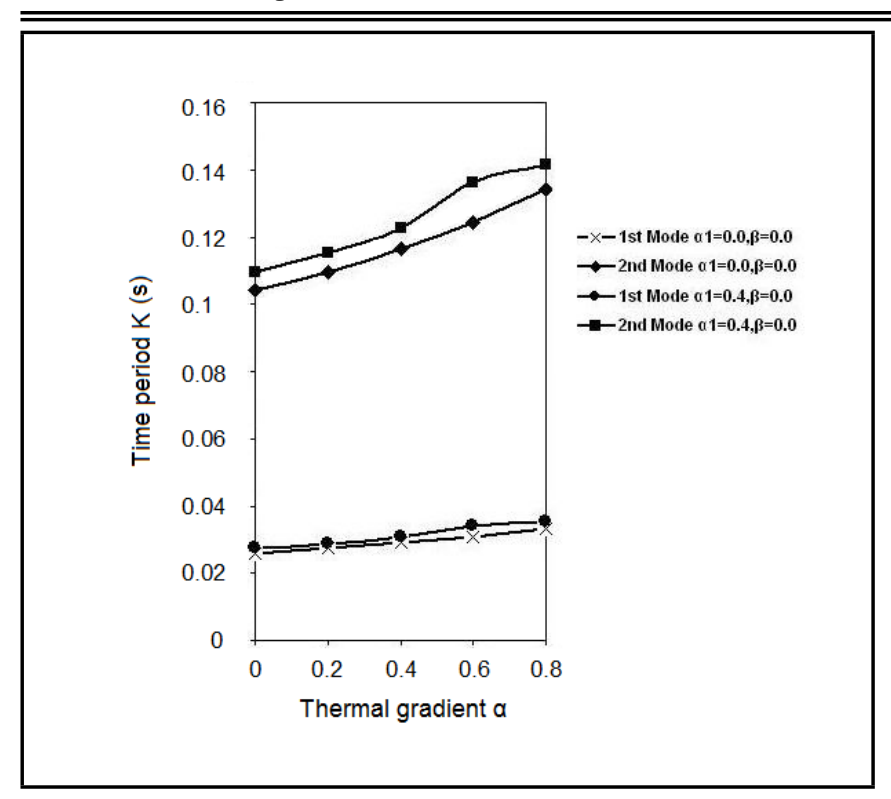

Figure 1. Variation of time period with thermal gradient of visco-elastic nonhomogeneous orthotropic rectangular plate of linearly varying thickness.

where

$$
k=\frac{\eta}{G}=\frac{\eta_{0}\left[1-\alpha_{4}(1-X)\right]}{G_{0}\left[1-\alpha_{5}(1-X)\right]} .
$$

Equation 28 is a differential equation of the second order for time function $T$. The solution of Eq. 28 will be

$$
T(t)=e^{a_{1} t}\left[C_{1} \cos b_{1} t+C_{2} \sin b_{1} t\right]
$$

where

$$
\begin{gathered}
a_{1}=-p^{2} m / 2 ; \\
b_{1}=p \sqrt{1-(p m / 2)^{2}} ;
\end{gathered}
$$

and $C_{1}, C_{2}$ are constants, which can be determined easily from the initial conditions of the plate. This study assumes the initial conditions as

$$
T=1 \quad \text { and } \quad T=0 \text { at } t=0 .
$$

Using Eq. 33 in Eq. 30, one obtains

$$
C_{1}=1 \quad \text { and } \quad C_{2}=-a_{1} / b_{1} .
$$

One has the equation

$$
T(t)=e^{a_{1} t}\left[\cos b_{1} t+\left(-a_{1} / b_{1}\right) \sin b_{1} t\right] ;
$$

after using Eq. 34 in Eq. 30. Thus, the deflection of the vibrating mode $w(x, y, t)$, which is equal to $W(x, y) T(t)$, may be expressed as

$$
\begin{aligned}
w= & {[X Y(a / b)(1-X)(1-Y a / b)]^{2} } \\
& {\left[1+\left(-b_{11} / b_{12}\right) X Y(a / b)(1-X)(1-Y a / b)\right] } \\
\times & {\left[e^{a_{1} t}\left\{\cos b_{1} t+\left(-a_{1} / b_{1}\right) \sin b_{1} t\right\}\right] ; }
\end{aligned}
$$

by using Eq. 24 and Eq. 35. The time period of the vibration of the plate is given by the following:

$$
K=2 \pi / p
$$

where $p$ is the frequency given by Eq. 23 .

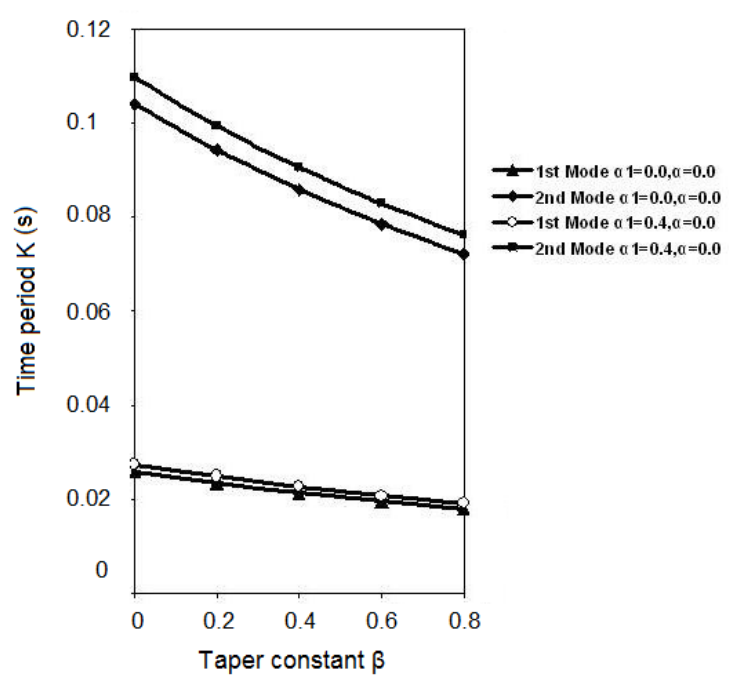

Figure 2. Variation of time period with taper constant of visco-elastic nonhomogeneous orthotropic rectangular plate of linearly varying thickness.

\section{NUMERICAL EVALUATIONS}

The values of the time period $(K)$ and the deflection $(w)$ (at two different instants of time) for a clamped visco-elastic orthotropic non-homogeneous rectangular plate for different values of the taper constant $\beta$, thermal gradients $\left(\alpha, \alpha_{4}, \alpha_{5}\right)$, the non-homogeneity constant $\alpha_{1}$, and the aspect ratio $a / b$ at different points for first two modes of vibrations have been calculated.

The following orthotropic material parameters have been taken as $^{2}$

- $E_{2}^{*} / E_{1}^{*}=0.32$

- $E^{*} / E_{1}^{*}=0.04$

- $G_{0} / E_{1}^{*}=0.09$

- $\eta_{0} / G_{0}=0.000069$

- $\rho_{0}=3 \times 10^{5}$ (mass density per unit volume of the plate material)

The thickness of the plate at the centre is taken as $h_{0}=$ 0.01 meter.

\section{RESULTS AND DISCUSSION}

The numerical results for a visco-elastic orthotropic nonhomogeneous rectangular plate of parabolically varying thickness have been computed with accuracy by using latest computer technology. Computations have been made for calculating the time period $K$ and deflection $W$ (at two different instants of time) for different values of the taper constant $\beta$, thermal constants $\left(\alpha, \alpha_{4}, \alpha_{5}\right)$, non-homogeneity constant $\alpha_{1}$, and the aspect ratio $a / b$ at different points for first two modes of vibration. All these results are presented in Figs. $1-8$. A comparison is made with the authors previous work ${ }^{3}$ for a uniform plate, and is found to be in very close agreement. 


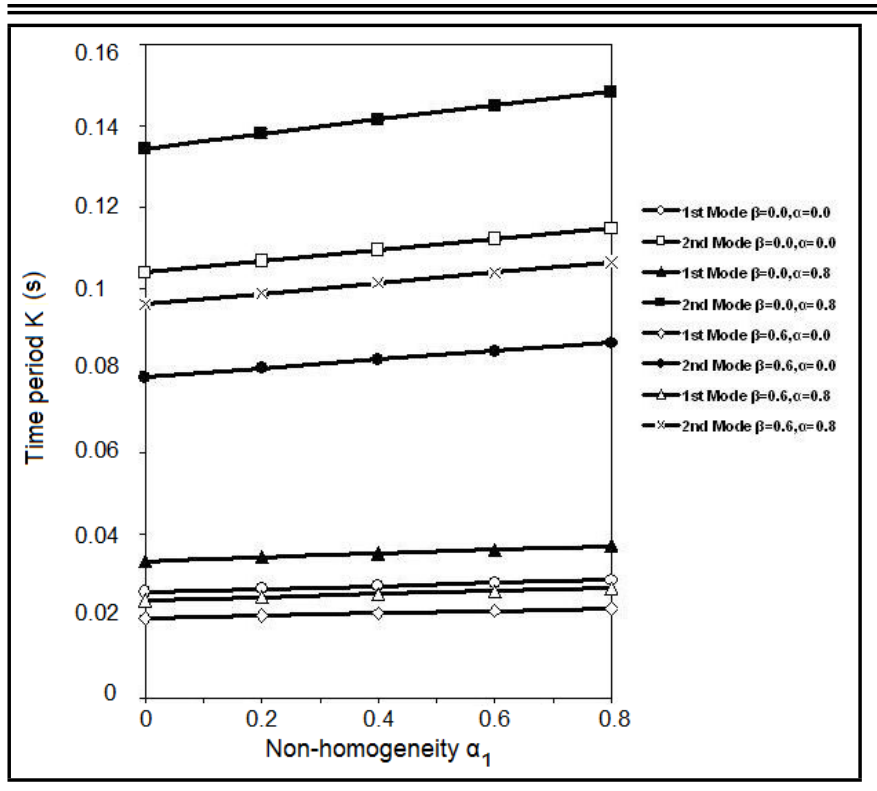

Figure 3. Variation of time period with non homogeneity constant of viscoelastic non-homogeneous orthotropic rectangular plate of linearly varying thickness.

- Figure 1 shows the results of the time period $(K)$ for different values of the thermal gradient $(\alpha)$, and the fixed taper constant $\beta=0.0$ and aspect ratio $a / b=1.5$ for two values of the non-homogeneity constant $\left(\alpha_{1}\right)$; i.e. $\alpha_{1}=0.0$ and $\alpha_{1}=0.4$ for the first two modes of vibration.

- Figure 2 shows the results of the time period $(K)$ for different values of the taper constant $(\beta)$, and the fixed thermal gradient $\alpha_{1}=0.0$ and aspect ratio $a / b=1.5$ for two values of the non-homogeneity constant $\left(\alpha_{1}\right)$; i.e. $\alpha_{1}=0.0$ and $\alpha_{1}=0.4$ for the first two modes of vibration.

- Figure 3 shows the results of the time period $(K)$ for first two modes of vibration for different values of the nonhomogeneity constant $\left(\alpha_{1}\right)$, aspect ratio $(=1.5)$, and four combinations of the taper constant $(\beta)$ and thermal gradient $(\alpha)$; i.e.

$\beta=0.0, \quad \alpha=0.0$

$\beta=0.0, \quad \alpha=0.8$

$\beta=0.6, \quad \alpha=0.0$

$\beta=0.6, \quad \alpha=0.8$

It can be seen that time period $(K)$ increases when the non-homogeneity constant $\left(\alpha_{1}\right)$ increases for first two modes of vibration.

- Figure 4 shows the results of the time period $(\mathrm{K})$ for different aspect ratios $(a / b)$, and four combinations of the thermal gradient $(\alpha)$, the taper constant $(\beta)$, and the nonhomogeneity constant $\left(\alpha_{1}\right)$; i.e.

$\alpha=0.8, \quad \beta=0.6, \quad \alpha_{1}=0.0$;

$\alpha=0.8, \quad \beta=0.6, \quad \alpha_{1}=0.4$;

$\alpha=0.0, \quad \beta=0.0, \quad \alpha_{1}=0.0$;

$\alpha=0.8, \quad \beta=0.0, \quad \alpha_{1}=0.4$

It can be seen that time period $(K)$ decreases when aspect ratio $(a / b)$ increases for first two modes of vibration.

- Figure 5-8 show the result of deflection for the first two

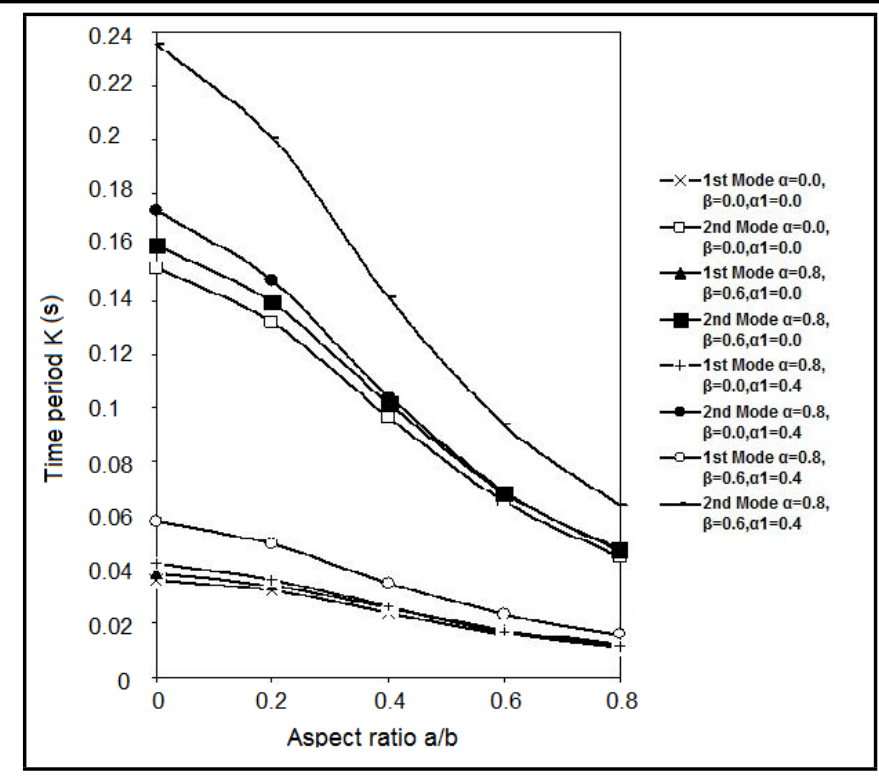

Figure 4. Variation of time period with non aspect ratio of visco-elastic nonhomogeneous orthotropic rectangular plate of linearly varying thickness.

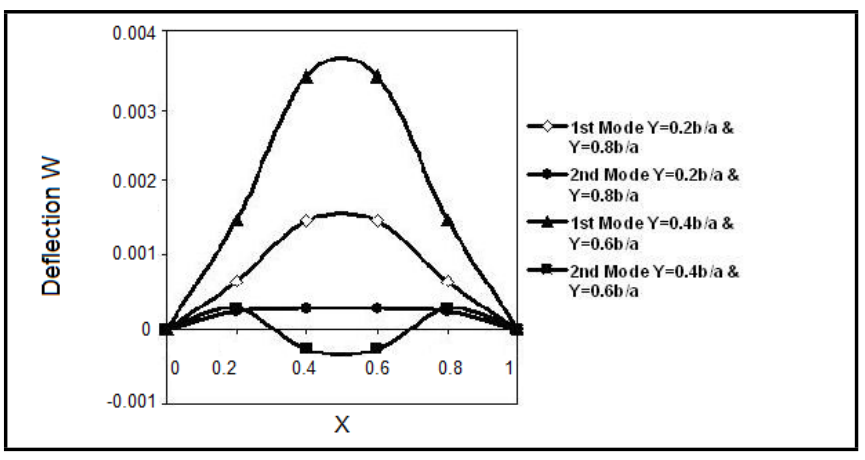

Figure 5. Deflection $w$ vs $X$ of visco-elastic non homogeneus orthotropic rectangular plate of linearly varying thickness at initial time $0 . \mathrm{K}$.

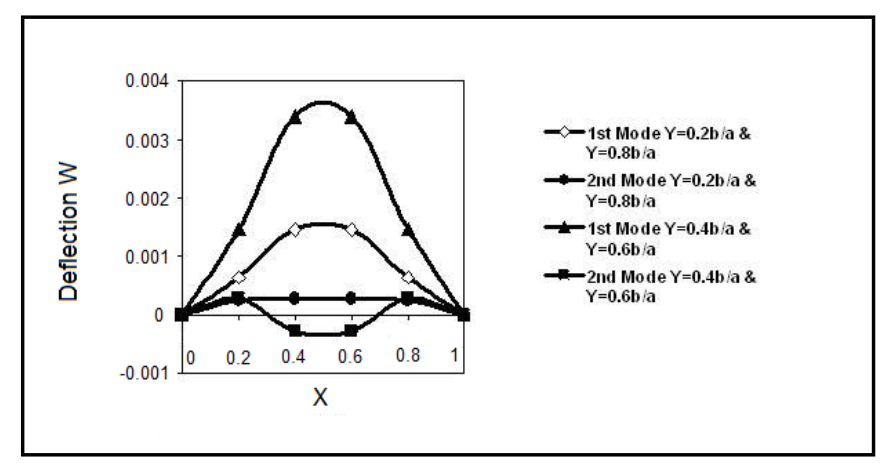

Figure 6. Deflection $w$ vs $X$ of visco-elastic non homogeneus orthotropic rectangular plate of linearly varying thickness at time $0 . \mathrm{K}$.

modes of vibration for different $X, Y$, and fixed aspect ratio $a / b=1.5$ for the initial time $0 . \mathrm{K}$ and time $5 . \mathrm{K}$ for the following combination of thermal gradients $\left(\alpha, \alpha_{4}, \alpha_{5}\right)$, the taper constant $\beta$, and the non-homogeneity constant $\alpha_{1}$.

- Figure 5: $\alpha=0.0, \quad \beta=0.6, \quad \alpha_{1}=\alpha_{4}=\alpha_{5}=0.0$, and the time is $0 . \mathrm{K}$.

- Figure 6: $\alpha=0.8, \quad \beta=0.6, \quad \alpha_{1}=\alpha_{4}=\alpha_{5}=0.0$, and the time is $0 . \mathrm{K}$. 


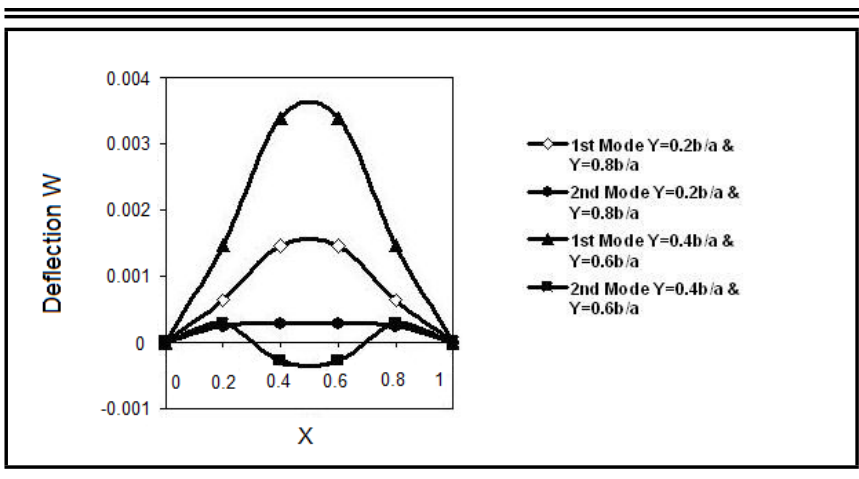

Figure 7. Deflection $w$ vs $X$ of visco-elastic non homogeneus orthotropic rectangular plate of linearly varying thickness at time 5.K.

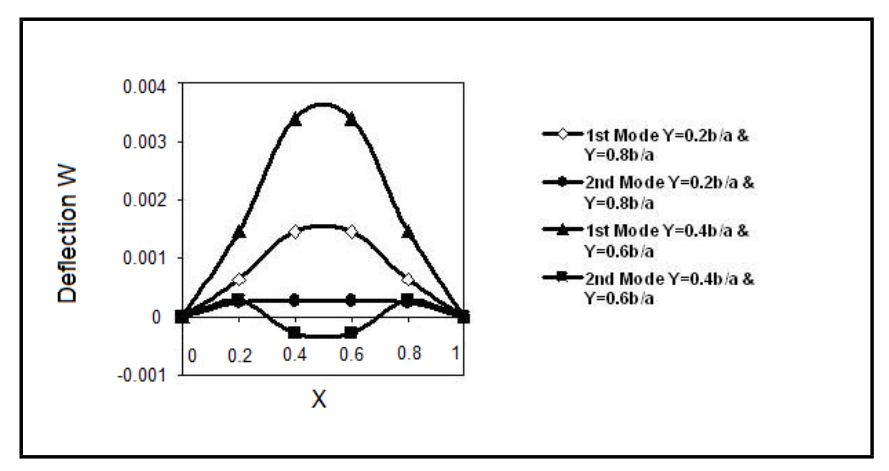

Figure 8. Deflection $w$ vs $X$ of visco-elastic non homogeneus orthotropic rectangular plate of linearly varying thickness at time $5 . \mathrm{K}$.

- Figure 7: $\alpha=0.0, \quad \beta=0.0, \quad \alpha_{1}=0.0, \quad \alpha_{4}=$ $0.3, \alpha_{5}=0.2$, and the time is $5 . \mathrm{K}$.

- Figure 8: $\alpha=0.8, \quad \beta=0.6, \quad \alpha_{1}=0.4, \quad \alpha_{4}=$ $0.3, \quad \alpha_{5}=0.2$, and the time is 5.K.

\section{REFERENCES}

1 Laura, P. A. A., Grossi, R. O., and Carneiro, G. I. Transverse vibrations of rectangular plates with thickness varying in two directions and with edges elastically restrained against rotation, Journal of Sound and Vibration, 63 (4), 499-505, (1979).

2 Leissa, A. W. NASA SP-60, Vibration of Plate, (1969).

3 Gupta, A. K. and Singhal, P. Effect of non-homogeneity on thermally induced vibration of orthotropic visco-elastic rectangular plate of linearly varying thickness, Applied Mathematics, 1 (4), 326-333, (2010).

4 Lal, R. Transverse vibrations of orthotropic non-uniform rectangular plate with continuously varying density, Indian Journal of Pure and Applied Mathematics, 34, 587-606, (2003).

5 Sobotka, Z. Free vibration of visco-elastic orthotropic rectangular plates, Acta Technica CSAV, 6, 678-705, (1978).

6 Singh, B. and Saxena, V. Transverse vibration of rectangular plate with bi-directional thickness variation, Journal of Sound and Vibration, 198, 51-65, (1996).
7 Bambill, D. V., Rossil, C. A., Laura, P. A. A., and Rossi, R. E. Transverse vibrations of an orthotropic rectangular plate of linearly varying thickness and with a free edge, Journal of Sound and Vibration, 235 (3), 530-538, (2000).

8 Tomar, J. S. and Gupta, A. K. Effect of thermal gradient on frequencies of orthotropic rectangular plate whose thickness varies in two directions, Journal of Sound and Vibration, 98 (2), 257-262, (1985).

9 Tomar, J. S. and Gupta, A. K. Thermal effect on frequencies of an orthotropic rectangular plate of linearly varying thickness, Journal of Sound and Vibration, 90 (3), 325-331, (1983).

10 Tomar, J. S. and Gupta, A. K. Effect of exponential temperature variation on frequencies of an orthotropic rectangular plate of exponentially varying thickness, Proceeding of the workshop on Computer Application in Continuum Mechanics, March 11-13, Depts. of Math. U.O.R, Roorkee, 183188, (1986).

11 Gupta, U. S., Lal, R., and Sharma, S. Vibration analysis of non-homogenous circular plate of nonlinear thickness variation by differential quadrature method, Journal of Sound and Vibration, 298 (4-5), 892-906, (2006).

12 Gupta, A. K. and Kumar, S. Effect of exponential temperature variation on vibration of orthotropic rectangular plate with linearly thickness variation in both directions, Pragyan-IT, IMS, Dehradun (India), 6 (2), 39-45, (2008).

13 Gupta, A. K., Johri, T., and Vats, R. P. Thermal effect on vibration of non-homogeneous orthotropic rectangular plate having bi-directional parabolically varying thickness, Proceeding of International Conference in World Congress on Engineering and Computer Science 2007 (WCECS 2007), San Francisco, USA ( 24-26 October, 2007), 784-787, (2007).

14 Gupta, A. K. and Khanna, A. Vibration of visco-elastic rectangular plate with linearly thickness variations in both directions, Journal of Sound and Vibration, 301 (3-5), 450457, (2007).

15 Laura, P. A. A. and Gutierrez, R. H. Vibration analysis on a rectangular plate subjected to a thermal gradient, Journal of Sound and Vibration, 72, 263-264, (1980).

16 Gupta, A. K. and Khanna, A. Vibration of clamped viscoelastic rectangular plate with parabolic thickness variations, Shock and Vibration, 15 (6), 713-723, (2008).

17 Gupta, A. K. and Kaur, H. Study of the effect of thermal gradient on free vibration of clamped visco-elastic rectangular plates with linearly thickness variation in both directions, Meccanica, 43, 449-458, (2008).

18 Gupta, A. K. and Kumar, S. Thermal effect on vibration of orthotropic rectangular plate with parabolic thickness variations, Acta Technica, 55, 315-325, (2010). 